\title{
Off-shell mean-field electromagnetic $T$-matrix of finite-size spheres and fuzzy scatterers
}

\author{
Yves-Patrick Pellegrini, D Brian Stout and Pascal Thibaudeau \\ Commissariat à l'Energie Atomique, Centre d'Etudes Scientifiques et Techniques d'Aquitaine,
} BP 2, 33114 Le Barp, France

Received 24 July 1996, in final form 26 September 1996

\begin{abstract}
We introduce a method for computing simple energy-conserving model $T$-matrices intended for use in multiple-scattering formalisms. Our method is to make separable approximations to the physical scattering potentials. Such a 'mean-field' approximation permits a ready analytic evaluation of the $T$-matrix for a variety of scatterers, even 'fuzzy' scatterers (e.g. fractal aggregates). In the small-size limit, our method reduces to the point-like resonant $T$-matrix introduced by Nieuwenhuizen et al in 1992 thereby demonstrating the intrinsic meanfield nature of their approximation. We discuss causality in both models and show that the separable potential model possesses a wider range of applicability than the point-like model does.
\end{abstract}

\section{Introduction}

The off-shell (i.e. momentum dependent) $T$-matrix of a scatterer [1-4] is of recognized importance in the study of wave propagation in random media [5]. The $T$-matrix, which solves the one-body problem for a homogeneous scatterer, is the building block of the well known multiple-scattering description of a composite random medium [4, 6-8]. Multiplescattering formalism is a powerful technique permitting the study of coherent and incoherent energy transfers in a medium built of discrete scatterers randomly dispersed in some background matrix. Various advances have recently been made in this field, fuelled by increasing interest in the possibility for localization of classical waves by disorder [4, 9]. The main application of the multiple-scattering formalism is the computation of various effective transport parameters characterizing the random medium on average.

Adding scatterers to a homogeneous medium amounts to introducing an additional (microscopic) length scale in the problem of wave propagation: the particle radius $a$. This in turn induces spatial dispersion, namely momentum dependence, in the various effective response kernels of interest. In particular, the self-energy kernels of the electromagnetic problem [10] are momentum dependent and determine, via dispersion relations, the normal coherent modes which can, theoretically, be excited in the system. Little is known about spatial dispersion in this context beyond the consequences associated with the fact that the bare mode (the mode that already propagates in the background homogeneous medium) is renormalized [9].

Recent theoretical predictions, based on a one-particle coherent potential approximation with spatial dispersion, nevertheless support the idea that at least two modes might be present in electromagnetic wave propagation (a successful observation has been reported for sound wave propagation) [11]. A predominance exchange of two solutions of the transverse 
dispersion relation for the mean electric field (one of which is the renormalized bare solution) has also been reported for a field theoretical model of continuum disorder [10]. As far as a multiple-scattering description of the random medium is concerned (e.g. in the research field of weak localization and enhanced back scattering), the possibility of several propagating modes is usually ignored (on account of the theoretical complications that it introduces). One of the problems is the explicit computation of the non-trivial modes. However, if the zeros of the dispersion relation are the roots of an analytic function of the momentum variable $k$, they may all be determined simultaneously in the upper complex $k$-plane as a function of the frequency by using a numerical implementation of Cauchy's theorem [12]. We recently applied such a method to a spatially dispersive dynamic generalization of the Clausius-Mossoti formula [13].

As far as analytical calculations of multiple scattering are concerned, one can hardly hope to go beyond a two-particle description of the medium [14]. Spatial dispersion then originates firstly from the momentum-dependent $T$-matrix itself and secondly from the two-point correlation function. All correlation functions must at least incorporate the exclusion-volume correction, which forbids scatterers to overlap. The exclusion effect and the momentum dependence of the $T$-matrix lead to spatially dispersive contributions of the same order of magnitude, since both are related to the same length scale $a$.

One of the obstacles to the calculation of the properties of a random medium is that momentum-dependent $T$-matrices are difficult to compute and handle. In order to avoid spatial dispersion in the self-energy at one-body order, but still preserve the physically important property of resonant scattering on the heterogeneities, Lagendijk and van Tiggelen [9] and Nieuwenhuizen et al [15] have introduced in the electromagnetic case a point-like (PL) resonant $T$-matrix, defined with the help of a momentum cut-off in the Fourier space. Its spatial support in the direct space is reduced to a point. One pitfall of this useful model is that one cannot guarantee the geometric consistency (and therefore the frequency dependence in the scattering regime) of any two-particle model built on it, as a separation distance still has to be introduced between particles in order to regularize the two-body integrals [14]. Moreover, as we show below, the PL model violates causality despite the fact that it obeys the unitarity identity [16] which expresses energy conservation during a scattering event (and from which one deduces the optical theorem).

In order to investigate more closely spatial dispersion effects in random media, and their possible connection with scattering, it therefore appears desirable to have at one's disposal a model $T$-matrix which does not suffer the above limitations, but which still is simple enough to remain manageable. We present here such a model, built on a separable potential approximation. We show that it amounts to performing a mean-field approximation on the inner fields. We furthermore demonstrate the mean-field nature of the PL model by deriving it in an appropriate small-size limit of the mean-field approximation.

We employ hereafter the $(2 \pi)^{3 / 2}$ symmetric convention for space Fourier transforms and work in the time harmonic regime, where time transforms are performed with weights and factors $\mathrm{d} t \exp (\mathrm{i} \omega t),[\mathrm{d} \omega \exp (-\mathrm{i} \omega t)] / 2 \pi$. Bold sans serif typefaces are used to denote dyadics such as the electric field Green function. MKSA units will be employed throughout this work.

\section{One-body problem and $T$-matrix of a scatterer}

In this section, we remind the reader of the Green function for the electric field originating from a point source in the presence of a single scatterer and thereby introduce the $T$-matrix. Let $\phi_{y}(\boldsymbol{r})$ denote the characteristic function of the scatterer, 'centred' at the point $\boldsymbol{y}$ (centre 
of mass, for instance). The function $\phi_{y}(\boldsymbol{r})$ is by definition equal to 1 if $\boldsymbol{r}$ lies within the scatterer and 0 otherwise (we shall see below that such a stiffness assumption may in fact be relaxed). Its volume integral is equal to the volume $V_{s}$ of the scatterer. Let also $\varepsilon_{m}$ and $\mu_{m}$ be the constitutive parameters of the outer medium, and $\varepsilon_{s}$ and $\mu_{s}$ those of the scatterer. The constitutive parameters of the whole system are then

$$
\begin{aligned}
\varepsilon(\boldsymbol{r}) & =\varepsilon_{m}+\left(\varepsilon_{s}-\varepsilon_{m}\right) \phi_{\boldsymbol{y}}(\boldsymbol{r}) \\
\frac{1}{\mu(\boldsymbol{r})} & =\frac{1}{\mu_{m}}+\left(\frac{1}{\mu_{s}}-\frac{1}{\mu_{m}}\right) \phi_{\boldsymbol{y}}(\boldsymbol{r}) .
\end{aligned}
$$

The related electric dyadic Green function is the outgoing wave solution of the electric field equation with a unit dyadic point source (I is the $3 \times 3$ identity matrix)

$$
\left(\nabla \times \frac{1}{\mu(\boldsymbol{r})} \nabla \times-\left(\frac{\omega}{c}\right)^{2} \varepsilon(\boldsymbol{r})\right) \mathbf{G}\left(\boldsymbol{r} \mid \boldsymbol{r}^{\prime}\right)=\mathbf{I} \delta^{3}\left(\boldsymbol{r}-\boldsymbol{r}^{\prime}\right) .
$$

Introducing the scattering potential $\mathbf{U}_{y}$

$\mathbf{U}_{y}\left(\boldsymbol{r} \mid \boldsymbol{r}^{\prime}\right)=\delta^{3}\left(\boldsymbol{r}-\boldsymbol{r}^{\prime}\right)\left[\boldsymbol{\nabla}^{\prime} \times\left(\frac{1}{\mu_{m}}-\frac{1}{\mu_{s}}\right) \phi_{y}\left(\boldsymbol{r}^{\prime}\right) \boldsymbol{\nabla}^{\prime} \times+\left(\frac{\omega}{c}\right)^{2}\left(\varepsilon_{s}-\varepsilon_{m}\right) \mathbf{I} \phi_{y}\left(\boldsymbol{r}^{\prime}\right)\right]$

the propagation equation (2.2) becomes

$$
\left(\frac{1}{\mu_{m}} \boldsymbol{\nabla} \times \nabla \times-\left(\frac{\omega}{c}\right)^{2} \varepsilon_{m}\right) \mathbf{G}\left(\boldsymbol{r} \mid \boldsymbol{r}^{\prime}\right)=\mathbf{I} \delta^{3}\left(\boldsymbol{r}-\boldsymbol{r}^{\prime}\right)+\int \mathrm{d}^{3} r_{1} \mathbf{U}_{y}\left(\boldsymbol{r} \mid \boldsymbol{r}_{1}\right) \mathbf{G}\left(\boldsymbol{r}_{1} \mid \boldsymbol{r}^{\prime}\right) .
$$

We denote the bare electric Green function for the outer medium by $\mathbf{G}_{0}$. In the Fourier representation it can be written $\mathbf{G}_{0}\left(\boldsymbol{k}_{1} \mid \boldsymbol{k}_{2}\right)=(2 \pi)^{3 / 2} \delta\left(\boldsymbol{k}_{1}-\boldsymbol{k}_{2}\right) \mathbf{G}_{0}\left(\boldsymbol{k}_{1}\right)$, where $\mathbf{G}_{0}\left(\boldsymbol{k}_{1}\right)$ is a linear combination of the transverse and longitudinal projectors defined in terms of the unit vector $\hat{k}=\boldsymbol{k} / k$

$$
\mathbf{G}_{0}(\boldsymbol{k})=\frac{1}{(2 \pi)^{3 / 2}}\left(\frac{\mu_{m}}{k^{2}-\left(k_{m}+\mathrm{i} 0\right)^{2}}[\mathbf{I}-\hat{\boldsymbol{k}} \hat{\boldsymbol{k}}]-\frac{1}{(\omega / c)^{2} \varepsilon_{m}} \hat{\boldsymbol{k}} \hat{\boldsymbol{k}}\right) .
$$

We have set $k_{m}^{2} \equiv(\omega / c)^{2} \varepsilon_{m} \mu_{m}$ and added an infinitesimal part to $k_{m}$ in the transverse part so as to select the outgoing-wave solution. Recasting (2.4) in integral form, we obtain

$$
\mathbf{G}\left(\boldsymbol{r} \mid \boldsymbol{r}^{\prime}\right)=\mathbf{G}_{0}\left(\boldsymbol{r} \mid \boldsymbol{r}^{\prime}\right)+\int \mathrm{d}^{3} r_{1} \mathrm{~d}^{3} r_{2} \mathbf{G}_{0}\left(\boldsymbol{r} \mid \boldsymbol{r}_{1}\right) \mathbf{U}_{y}\left(\boldsymbol{r}_{1} \mid \boldsymbol{r}_{2}\right) \mathbf{G}\left(\boldsymbol{r}_{2} \mid \boldsymbol{r}^{\prime}\right) .
$$

The translation of (2.6) to the Fourier representation only requires the replacing of the position variables $r$ by the momentum coordinates $\boldsymbol{k}$. We may therefore use the simpler symbolic operator notation $G=G_{0}+G_{0} U_{y} G$, where we abandon the sans serif typefaces. In this notation the convolution identity $\mathbf{I} \delta^{3}\left(\boldsymbol{r}-\boldsymbol{r}^{\prime}\right)$ becomes 1 .

In the Fourier representation, the scattering potential is

$$
\begin{aligned}
\mathbf{U}_{\boldsymbol{y}}\left(\boldsymbol{k}_{1} \mid \boldsymbol{k}_{2}\right)= & \frac{\exp \left[\mathrm{i}\left(\boldsymbol{k}_{2}-\boldsymbol{k}_{1}\right)\right] \cdot \boldsymbol{y}}{(2 \pi)^{3 / 2}}\left\{\left(\frac{1}{\mu_{s}}-\frac{1}{\mu_{m}}\right) \boldsymbol{k}_{1} \times \boldsymbol{k}_{2} \times+\left(\frac{\omega}{c}\right)^{2}\left(\varepsilon_{s}-\varepsilon_{m}\right) \mathbf{I}\right\} \\
& \times \phi_{\mathbf{0}}\left(\boldsymbol{k}_{1}-\boldsymbol{k}_{2}\right) .
\end{aligned}
$$

This formulation automatically takes into account the boundary conditions on the fields on the surface of the scatterer [17]. The Fourier transform $\phi_{\mathbf{0}}(\boldsymbol{k})$ is proportional to the volume of the scatterer. It is purely real for bodies with a symmetry centre, whence $\mathbf{U}_{\mathbf{0}}\left(\boldsymbol{k}_{1} \mid \boldsymbol{k}_{2}\right)=\mathbf{U}_{\mathbf{0}}\left(-\boldsymbol{k}_{1} \mid-\boldsymbol{k}_{2}\right)$. For a sphere of finite radius $a, \phi_{\mathbf{0}}(\boldsymbol{r})=\theta(a-|\boldsymbol{r}|)$. Here and in the rest of the paper, the bold-face subscript $\mathbf{0}$ stands for $\boldsymbol{y}=\mathbf{0}$ (this remark applies to $\phi_{y}, \mathbf{U}_{y}$ and $\mathbf{T}_{y}$ ). 
The $T$-matrix of the scatterer, namely $T_{y}$, is defined by the equation [1]

$$
G=G_{0}+G_{0} T_{y} G_{0}
$$

which distinguishes between the contributions of the incident and scattered fields to the total field. Its expression in terms of the scattering potential $U$ and of the free Green function $G_{0}$ follows:

$$
T_{y}=U_{y}\left(1-G_{0} U_{y}\right)^{-1} .
$$

The difficult task in this formulation is the inversion of the operator $1-G_{0} U_{y}$. In the Fourier domain, $\mathbf{T}_{\boldsymbol{y}}\left(\boldsymbol{k}_{1} \mid \boldsymbol{k}_{2}\right)=\exp \left[\mathrm{i}\left(\boldsymbol{k}_{2}-\boldsymbol{k}_{1}\right) \cdot \boldsymbol{y}\right] \mathbf{T}_{\mathbf{0}}\left(\boldsymbol{k}_{1} \mid \boldsymbol{k}_{2}\right)$. The support of $\mathbf{T}_{\boldsymbol{y}}\left(\boldsymbol{r}_{1} \mid \boldsymbol{r}_{2}\right)$ is the volume of the scatterer, for each of its two arguments. To see this, we realize that this property is obeyed by $\mathbf{U}_{y}\left(\boldsymbol{r}_{1} \mid \boldsymbol{r}_{2}\right)$ and, in consequence, by each term of the series expansion of (2.9), since they all begin and end with a $U_{y}$ [7].

According to equation (2.8), knowing the fully momentum-dependent $T$-matrix of a given scatterer is equivalent to knowing simultaneously the solutions of the inner, outer and mixed scattering problems for arbitrary positions of the source and the receiver. Both may be located either inside or outside the scatterer.

\section{The mean-field $T$-matrix}

Our model follows from a modification of the constitutive relations (2.1) so that the formal expansion of the $T$-matrix $T_{0}=G_{0}^{-1} \sum_{p \geqslant 1}\left(G_{0} U_{0}\right)^{p}$ deduced from (2.9) reduces to an algebraic geometrical series. The series is then straightforwardly summed. The modification resides in a shift from locality to non-locality in the constitutive relations, so that the scattering potential becomes separable in its two space variables. That is, we replace the true constitutive relations, equation (2.1), by the approximations

$$
\begin{aligned}
& \varepsilon(\boldsymbol{r}) \delta^{3}\left(\boldsymbol{r}-\boldsymbol{r}^{\prime}\right) \rightarrow \varepsilon\left(\boldsymbol{r} \mid \boldsymbol{r}^{\prime}\right)=\varepsilon_{m}\left[\delta^{3}\left(\boldsymbol{r}-\boldsymbol{r}^{\prime}\right)+\Delta \varepsilon \frac{1}{V_{s}} \phi_{\boldsymbol{y}}(\boldsymbol{r}) \phi_{y}\left(\boldsymbol{r}^{\prime}\right)\right] \\
& \frac{1}{\mu}(\boldsymbol{r}) \delta^{3}\left(\boldsymbol{r}-\boldsymbol{r}^{\prime}\right) \rightarrow \mu^{-1}\left(\boldsymbol{r} \mid \boldsymbol{r}^{\prime}\right)=\frac{1}{\mu_{m}}\left[\delta^{3}\left(\boldsymbol{r}-\boldsymbol{r}^{\prime}\right)+\Delta \mu \frac{1}{V_{s}} \phi_{\boldsymbol{y}}(\boldsymbol{r}) \phi_{\boldsymbol{y}}\left(\boldsymbol{r}^{\prime}\right)\right]
\end{aligned}
$$

where $V_{s}$ is the volume of the scatterer, $\Delta \varepsilon=\varepsilon_{s} / \varepsilon_{m}-1$, and $\Delta \mu=\mu_{m} / \mu_{s}-1$.

The electric displacement is obtained from the formula $\boldsymbol{D}(\boldsymbol{r})=\varepsilon_{0} \int \mathrm{d}^{3} \boldsymbol{r}^{\prime} \varepsilon\left(\boldsymbol{r} \mid \boldsymbol{r}^{\prime}\right) \boldsymbol{E}\left(\boldsymbol{r}^{\prime}\right)$. A similar relation holds between the magnetic induction $\boldsymbol{H}$ and the magnetic field $\boldsymbol{B}$. Hence if $\boldsymbol{r}$ is outside the scatterer, $\boldsymbol{D}(\boldsymbol{r})=\varepsilon_{0} \varepsilon_{m} \boldsymbol{E}(\boldsymbol{r})$ as usual while, for $\boldsymbol{r}$ inside,

$$
\boldsymbol{D}(\boldsymbol{r})=\varepsilon_{0} \varepsilon_{m}\left(\boldsymbol{E}(\boldsymbol{r})-\frac{1}{V_{s}} \int_{V_{s}} \mathrm{~d}^{3} \boldsymbol{r}^{\prime} \boldsymbol{E}\left(\boldsymbol{r}^{\prime}\right)\right)+\varepsilon_{0} \varepsilon_{s} \frac{1}{V_{s}} \int_{V_{s}} \mathrm{~d}^{3} \boldsymbol{r}^{\prime} \boldsymbol{E}\left(\boldsymbol{r}^{\prime}\right)
$$

instead of being $\boldsymbol{D}(\boldsymbol{r})=\varepsilon_{0} \varepsilon_{s} \boldsymbol{E}(\boldsymbol{r})$. The spatially varying part of the displacement field inside the scatterer becomes proportional to $\varepsilon_{m}$ in (3.2). The dielectric mismatch is taken into account only through volume averages of the inner fields. This relegates the model to the wide class of mean-field theories. We shall therefore refer to the $T$-matrix obtained from the separable potential as the mean-field approximation. This approximation, as it stands, is not able to restore the dynamic behaviour of the fields inside the scatterer when $\varepsilon_{s}$ differs appreciably from $\varepsilon_{m}$. Indeed, the inner wavevector modulus is clearly always pinned at $k_{m}$. However, since the remaining calculations may in principle be carried out exactly, the resulting $T$-matrix automatically obeys energy conservation, in accordance with the unitarity identity [16]

$$
T_{y}-T_{y}^{\dagger}=T_{y}^{\dagger}\left(G_{0}-G_{0}^{\dagger}\right) T_{y}=T_{y}\left(G_{0}-G_{0}^{\dagger}\right) T_{y}^{\dagger}
$$


with the daggers representing Hermitian conjugation. This identity holds provided that both the inner and the outer media are non-dissipative, i.e. when the scattering potential is Hermitian: $U_{y}=U_{y}^{\dagger}$. Scatterers for which $y$ is a symmetry centre obey the property, inherited from $U_{\mathbf{0}}$, that $\mathbf{T}_{\mathbf{0}}\left(-\boldsymbol{k}_{1} \mid-\boldsymbol{k}_{2}\right)=\mathbf{T}_{\mathbf{0}}\left(\boldsymbol{k}_{1} \mid \boldsymbol{k}_{2}\right)$. The above unitary identity then is

$$
\operatorname{Im}\left[\mathbf{T}_{\mathbf{0}}\left(\boldsymbol{k}_{1} \mid \boldsymbol{k}_{2}\right)\right]=\frac{\pi}{2} \mu_{m} k_{m} \int \mathrm{d} \Omega_{q} \mathbf{T}_{\mathbf{0}}\left(\boldsymbol{k}_{1} \mid k_{m} \hat{\boldsymbol{q}}\right)[\mathbf{I}-\hat{\boldsymbol{q}} \hat{\boldsymbol{q}}] \mathbf{T}_{\mathbf{0}}^{*}\left(k_{m} \hat{\boldsymbol{q}} \mid \boldsymbol{k}_{2}\right)
$$

where $\int \mathrm{d} \Omega_{q}$ indicates an angular integration carried out over the unit vector $\hat{\boldsymbol{q}}$ and the asterisk represents complex conjugation.

The Fourier transform of the scattering potential $U_{\mathbf{0}}$ obtained from the separable approximation to the constitutive relations, equations (3.1) is

$$
\mathbf{U}_{\mathbf{0}}\left(\boldsymbol{k}_{1} \mid \boldsymbol{k}_{2}\right)=\frac{V_{s}}{\mu_{m}}\left(\Delta \mu \boldsymbol{k}_{1} \times \boldsymbol{k}_{2} \times+k_{m}^{2} \Delta \varepsilon \mathbf{I}\right) \psi_{\mathbf{0}}\left(\boldsymbol{k}_{1}\right) \psi_{\mathbf{0}}^{*}\left(\boldsymbol{k}_{2}\right)
$$

where we have defined $\psi_{0} \equiv \phi_{0} / V_{s}$, in order to extract the explicit proportionality of $\phi_{\mathbf{o}}(\boldsymbol{k})$ to $V_{s}$. We remark that, in the direct representation, owing to the double multiplier $\psi_{0}\left(\boldsymbol{r}_{1}\right) \psi_{\mathbf{0}}\left(\boldsymbol{r}_{2}\right)$, the support of the mean-field scatterer for each of the two variables $\boldsymbol{r}_{1}, \boldsymbol{r}_{2}$, is still the original object (although it is considerably enlarged in the product space of the variables $\boldsymbol{r}_{1}$ and $\boldsymbol{r}_{2}$ ). This ensures the correct implementation of geometric exclusion effects between different scatterers.

In general, except for a sphere, $\psi_{\mathbf{0}}(\boldsymbol{q})$ has no spherical symmetry. For example, it depends on a scalar combination of the type $\boldsymbol{q} \cdot \mathbf{O} \boldsymbol{q}$ for an ellipsoid. Such a lack of spherical symmetry complicates the evaluation of $\left(G_{0} U_{\mathbf{o}}\right)^{p}$ by coupling the dielectric and the magnetic parts. In the mean-field treatment, the couplings vanish in the case of spherical symmetry. We may therefore further simplify the model (on a heuristic basis) by replacing $\psi_{0}(q)$ by its symmetrized angular average $\overline{\psi_{0}}(q)=\int \mathrm{d} \Omega_{q} \psi_{0}(\boldsymbol{q}) / 4 \pi$. We remark in passing that $\psi_{0}(\boldsymbol{q})$ is purely real, and that the angular averaging operator - commutes with the threedimensional Fourier transform. We shall term this approximation the mean-field spherical (MFS) approximation.

The average $\overline{\psi_{0}}(r)$ represents a fuzzy scatterer with soft boundaries instead of the original anisotropic stiff object. Nevertheless, $\overline{\psi_{0}}(r)$ still possesses a compact support. After some easy algebra we find for $p \geqslant 1$, that

$$
\begin{aligned}
&\left(\mathbf{G}_{0} \mathbf{U}_{\mathbf{0}}\right)^{p}\left(\boldsymbol{k}_{1} \mid \boldsymbol{k}_{2}\right)=(2 \pi)^{3 / 2} \frac{V_{s}}{\mu_{m}} \mathbf{G}_{0}\left(\boldsymbol{k}_{1}\right)\left[\Delta \mu \boldsymbol{k}_{1} \times(\Delta \mu \mathbf{M})^{p-1} \boldsymbol{k}_{2} \times+k_{m}^{2} \Delta \varepsilon(\Delta \varepsilon \mathbf{E})^{p-1}\right] \\
& \times \overline{\psi_{\mathbf{0}}}\left(k_{1}\right) \overline{\psi_{\mathbf{0}}}\left(k_{2}\right)
\end{aligned}
$$

where the dyadics $\mathbf{M}$ and $\mathbf{E}$ are defined as

$$
\begin{aligned}
& \mathbf{E} \equiv(2 \pi)^{3 / 2} \frac{V_{s}}{\mu_{m}} k_{m}^{2} \int \mathrm{d}^{3} q{\overline{\psi_{0}}}^{2}(q) \mathbf{G}_{0}(\boldsymbol{q}) \\
& \mathbf{M} \equiv(2 \pi)^{3 / 2} \frac{V_{s}}{\mu_{m}} \int \mathrm{d}^{3} q{\overline{\psi_{\mathbf{0}}}}^{2}(q) \boldsymbol{q} \times \mathbf{G}_{0}(\boldsymbol{q}) \boldsymbol{q} \times .
\end{aligned}
$$

Summing the geometrical series $\sum_{p \geqslant 1}\left(G_{0} U_{\mathbf{0}}\right)^{p}$ and pre-multiplying the result by $G_{0}^{-1}$, we obtain finally

$$
\begin{aligned}
\mathbf{T}_{\mathbf{0}}\left(\boldsymbol{k}_{1} \mid \boldsymbol{k}_{2}\right)= & \frac{V_{s}}{\mu_{m}}\left\{\boldsymbol{k}_{1} \times \Delta \mu[1-\Delta \mu \mathbf{M}]^{-1} \boldsymbol{k}_{2} \times+k_{m}^{2} \Delta \varepsilon[1-\Delta \varepsilon \mathbf{E}]^{-1}\right\} \\
& \times \overline{\psi_{\mathbf{0}}}\left(k_{1}\right) \overline{\psi_{\mathbf{0}}}\left(k_{2}\right) .
\end{aligned}
$$

In order to compute $\mathbf{E}$ and $\mathbf{M}$, we recall that by definition $V_{s}=\int \mathrm{d}^{3} r \phi_{\mathbf{0}}(\boldsymbol{r})=\int \mathrm{d}^{3} r \overline{\phi_{\mathbf{0}}}(r)$. By Parseval's theorem, $\int \mathrm{d}^{3} q{\overline{\psi_{0}}}^{2}(q)=\int \mathrm{d}^{3} r{\overline{\phi_{\mathrm{o}}}}^{2}(r) / V_{s}^{2}$. Since $\overline{\phi_{0}}(r) \leqslant 1$, we may write 
$\int \mathrm{d}^{3} r{\overline{\phi_{0}}}^{2}(r)=\lambda_{f} \int \mathrm{d}^{3} r \overline{\phi_{0}}(r)=\lambda_{f} V_{s}$, where $0<\lambda_{f} \leqslant 1$ is a numerical coefficient which measures the degree of fuzziness (or of anisotropy) of the scatterer. Therefore, $\int \mathrm{d}^{3} q{\overline{\psi_{0}}}^{2}(q)=\lambda_{f} / V_{s}$. For a perfect sphere, the identity $\phi_{0}(r)=\phi_{0}^{2}(r)$ entails $\lambda_{f}=1$.

Carrying out the integration over angles first in equations (3.7) amounts to taking the trace of the dyadics and multiplying by $4 \pi / 3$. We readily obtain

$$
\begin{aligned}
& \mathbf{E}=-\frac{1}{3} V_{s}\left(\frac{\lambda_{f}}{V_{s}}-2 k_{m}^{2} K\right) \mathbf{I} \\
& \mathbf{M}=-\frac{2}{3} V_{s}\left(\frac{\lambda_{f}}{V_{s}}+k_{m}^{2} K\right) \mathbf{I}
\end{aligned}
$$

where $K$ is

$$
\begin{aligned}
K & \equiv \int \mathrm{d}^{3} q \frac{{\overline{\psi_{0}}}^{2}(q)}{q^{2}-\left(k_{m}+\mathrm{i} 0\right)^{2}} \\
& =\int \mathrm{d}^{3} r \frac{\mathrm{e}^{\mathrm{i} k_{m} r}}{4 \pi r} p(r) .
\end{aligned}
$$

In the last line we introduced the normalized distribution

$$
p(r)=\int d^{3} R \overline{\psi_{0}}(|\boldsymbol{r}-\boldsymbol{R}|) \overline{\psi_{\mathbf{0}}}(R)
$$

interpreted as the probability distribution for finding two points within the scatterer separated by a distance $r$ (this follows since $\overline{\psi_{0}}(r)$ is defined as a symmetrized probability density for $r$ to belong to the scatterer).

Introducing effective dynamic dielectric and magnetic depolarization coefficients given by

$$
\bar{L}_{\varepsilon}=\frac{1}{3}\left[\lambda_{f}-2 k_{m}^{2} V_{s} K\right]
$$

and

$$
\bar{L}_{\mu}=\frac{1}{3}\left[\left(3-2 \lambda_{f}\right)-2 k_{m}^{2} V_{s} K\right]
$$

respectively, we find in the MFS approximation that

$\mathbf{T}_{\boldsymbol{y}}\left(\boldsymbol{k}_{1} \mid \boldsymbol{k}_{2}\right)=\frac{V_{s}}{\mu_{m}} \exp \left[\mathrm{i}\left(\boldsymbol{k}_{2}-\boldsymbol{k}_{1}\right) \cdot \boldsymbol{y}\right]\left\{-\bar{\alpha}_{\mu} \boldsymbol{k}_{1} \times \boldsymbol{k}_{2} \times+k_{m}^{2} \bar{\alpha}_{\varepsilon} \mathbf{I}\right\} \overline{\Psi_{\mathbf{0}}}\left(k_{1}\right) \overline{\boldsymbol{\psi}_{\mathbf{0}}}\left(k_{2}\right)$.

The effective dynamic polarizabilities $\bar{\alpha}_{\varepsilon}$ and $\bar{\alpha}_{\mu}$ are

$$
\begin{aligned}
& \bar{\alpha}_{\varepsilon}=\frac{\varepsilon_{s}-\varepsilon_{m}}{\varepsilon_{m}+\bar{L}_{\varepsilon}\left(\varepsilon_{s}-\varepsilon_{m}\right)} \\
& \bar{\alpha}_{\mu}=\frac{\mu_{s}-\mu_{m}}{\mu_{m}+\bar{L}_{\mu}\left(\mu_{s}-\mu_{m}\right)} .
\end{aligned}
$$

It should be clear that the previous method also applies to intrinsically fuzzy scatterers for which only a statistical description is available a priori, e.g. fractal aggregates [18]. Such objects will fit in a multiple-scattering theory of well separated aggregates each of finite extension, i.e. being described by $\psi_{0}(r)$ functions possessing compact support. In such a theory, one particular aggregate would be considered as a single scattering unit.

It is interesting to remark that equation (3.14a) has previously surfaced in the literature [19] as a phenomenological effective static scalar dielectric polarizability formula for ellipsoids. An effective static depolarization coefficient $\bar{L}$ was defined by averaging the eigenvalues of the ellipsoidal dielectric tensor:

$$
\frac{\varepsilon_{s}-\varepsilon_{m}}{\varepsilon_{m}+\bar{L}\left(\varepsilon_{s}-\varepsilon_{m}\right)} \equiv \frac{1}{3} \sum_{i=1}^{3} \frac{\varepsilon_{s}-\varepsilon_{m}}{\varepsilon_{m}+L_{i}\left(\varepsilon_{s}-\varepsilon_{m}\right)}
$$


where the $L_{i}$ are the three eigenvalues of the depolarization tensor for ellipsoids, linked by $\sum_{i} L_{i}=1$ [20]. The coefficient $\bar{L}$ depends in general on $\varepsilon_{s}$ and $\varepsilon_{m}$. Hence, the use of $\bar{L}$ as a purely geometrical parameter is valid only in limiting cases, such as weak dielectric contrast and small eccentricity (see [19] for further details). For anisotropic scatterers, it is clear that the same restrictions apply to our model as well. In the above calculation, however, $\bar{L}_{\varepsilon, \mu}$ relates not only to anisotropy but also more generally to the concept of fuzziness. This may enlarge the number of possible interpretations of experimental results that might be fitted with the above polarizability formula.

For a stiff sphere with radius $a$, one has $\psi_{\mathbf{0}}(\boldsymbol{q})=3(2 \pi)^{-3 / 2} j_{1}(a q) /(a q)$, and the meanfield spherical approximation coincides with the mean-field approximation. The $K$ integral can then be directly computed from $(3.10 a)$, yielding

$$
\bar{L}_{\varepsilon, \mu}=1-2 \mathrm{i}\left(a k_{m}\right) h_{1}^{(1)}\left(a k_{m}\right) j_{1}\left(a k_{m}\right) \text {. }
$$

\section{The point-like limit}

We may perform an asymptotic evaluation of integral (3.10a) when the size of the scatterer goes to zero. The distribution $p(r)$, of support twice as large as that of $\overline{\psi_{0}}(r)$, is highly peaked at $r=0$ when the support of $\overline{\psi_{\mathrm{o}}}(r)$ shrinks. We may then expand $\exp \left(\mathrm{i} k_{m} r\right) / r=1 / r+\mathrm{i} k_{m}+\mathrm{O}(r)$ under the integral and integrate term by term. Only the first two terms survive and we find that

$$
K \simeq \frac{1}{4 \pi}\left(\frac{1}{a_{p}}+\mathrm{i} k_{m}\right)
$$

where we defined $1 / a_{p} \equiv \int \mathrm{d}^{3} r p(r) / r$. This term goes to infinity in the PL limit. As discussed in [9], this divergence indicates that a point does not scatter waves. For a fuzzy scatterer, we also define a mean spherical radius $a$ according to $a^{3} \equiv 3 V_{s} / 4 \pi$ and introduce a dimensionless coefficient $\gamma_{f}=a / a_{p}$. The PL scatterer is then characterized by the depolarization coefficients

$$
\begin{aligned}
& \bar{L}_{\varepsilon}=\frac{\lambda_{f}}{3}-\frac{2}{9} \gamma_{f}\left(a k_{m}\right)^{2}-\frac{2}{9} \mathrm{i}\left(a k_{m}\right)^{3} \\
& \bar{L}_{\mu}=\frac{3-2 \lambda_{f}}{3}-\frac{2}{9} \gamma_{f}\left(a k_{m}\right)^{2}-\frac{2}{9} \mathrm{i}\left(a k_{m}\right)^{3} .
\end{aligned}
$$

To summarize, taking the PL limit in this manner amounts to truncating the series expansion of $\bar{L}_{\varepsilon, \mu}$ at third order. The justification for a truncation at precisely third order is provided by our focusing on the electromagnetic integrals alone in (3.7), leaving aside the pre-factor $V_{s}$ of geometrical nature. In this way, we are able to isolate the terms physically relevant (i.e. the non-zero terms) to electrodynamics in the PL limit; these are the first three terms in a wavevector expansion of (3.7), the first two terms being divergent and shapedependent (i.e. involving $\lambda_{f}$ and $\gamma_{f}$ ), and the third being finite and purely imaginary. Then, multiplying by the pre-factor $V_{s}$ to obtain the final results, we implicitly revert to a finitesized description, thereby 'stopping the shrinking process', in order to ensure a dynamic coupling of the object to the incident wave. In this sense, the surviving terms in (4.1) are minimal-coupling terms. We notice that the purely imaginary term in the PL limit, $\mathrm{i}\left(a k_{m}\right)^{3}$ possesses a somewhat universal character since it does not depend on the precise shape of the scatterer (because the definition of $a$ involves only the volume of the scatterer).

Note that, in the PL limit, one also has $\overline{\psi_{0}}\left(k_{1}\right) \overline{\psi_{0}}\left(k_{2}\right) \equiv(2 \pi)^{-3}$ in equation (3.13). Then $\mathbf{T}_{\boldsymbol{y}}\left(\boldsymbol{r}_{1} \mid \boldsymbol{r}_{2}\right) \propto \delta^{3}\left(\boldsymbol{r}_{1}-\boldsymbol{y}\right) \delta^{3}\left(\boldsymbol{r}_{2}-\boldsymbol{y}\right)$ and justifies the term 'point-like'. We may equally verify 
that this limit preserves the unitarity identity, equation (3.4). For the mean-field spherical model, this identity is easily shown to be equivalent to

$$
\operatorname{Im} \bar{\alpha}_{\varepsilon, \mu}=\frac{2(2 \pi)^{3}}{9}\left(a k_{m}\right)^{3}\left|\bar{\alpha}_{\varepsilon, \mu}\right|^{2} \overline{\psi_{0}}\left(k_{m}\right)^{2}
$$

which reduces to

$$
\operatorname{Im} \bar{L}_{\varepsilon, \mu}=-\frac{2}{9}\left(a k_{m}\right)^{3}(2 \pi)^{3}{\overline{\psi_{0}}}^{2}\left(k_{m}\right) .
$$

Since in the PL limit we find that $\operatorname{Im} \bar{L}_{\varepsilon, \mu}=-\frac{2}{9}\left(a k_{m}\right)^{3}$, equation (4.4) implies $\overline{\psi_{0}}(k) \equiv$ $(2 \pi)^{-3 / 2}$ in order to satisfy energy conservation. In this limit, equation (4.3) reduces to the familiar optical theorem relation applied to scalar polarizabilities [9].

Similar (although less precise) conclusions were arrived at in an earlier version of this work [21] by setting $\phi_{\mathbf{o}}(\boldsymbol{k}) \equiv(2 \pi)^{-3 / 2} V_{s}$ from the start, namely equation (2.7), and applying a well known device of statistical field theory [22] which is to regularize the now ultraviolet-diverging Fourier integrals (3.7) with a large cut-off $\Lambda$ on the inner momentum $q$. This was actually the original method used in [15]. Through an identification $\int_{q \leqslant \Lambda} \mathrm{d}^{3} q=(2 \pi)^{3} / V_{s}$, this faster 'trick' leads to (4.2) with the specific shape parameters $\lambda_{f}=1$ and $\gamma_{f}=9(9 \pi / 2)^{-2 / 3} \simeq 1.54$. We have clearly demonstrated above that the cut-off method belongs to the class of mean-field calculations, a fact which is not apparent in [15].

For real dielectric and magnetic constitutive parameters in the PL approximation, we find that resonant dielectric and magnetic scattering occur for the frequencies satisfying

$$
\begin{aligned}
\left(a k_{m}\right)_{\varepsilon}^{2} & =\frac{3}{2 \gamma_{f}} \frac{\lambda_{f} \varepsilon_{s}+\left(3-\lambda_{f}\right) \varepsilon_{m}}{\varepsilon_{s}-\varepsilon_{m}} \\
\left(a k_{m}\right)_{\mu}^{2} & =\frac{3}{2 \gamma_{f}} \frac{\left(3-2 \lambda_{f}\right) \mu_{s}+2 \lambda_{f} \mu_{m}}{\mu_{s}-\mu_{m}} .
\end{aligned}
$$

respectively. We may define resonance widths for $\bar{\alpha}_{\varepsilon}$ and $\bar{\alpha}_{\mu}$ of order $\frac{2}{9}\left(a k_{m}\right)_{\varepsilon}^{3}\left(\varepsilon_{s}-\varepsilon_{m}\right)$ and $\frac{2}{9}\left(a k_{m}\right)_{\mu}^{3}\left(\mu_{s}-\mu_{m}\right)$, respectively. Assuming positive constitutive parameters, this model displays resonant behaviour only if $\varepsilon_{s}>\varepsilon_{m}$ and $\mu_{s}>\mu_{m}$.

In the separable potential treatment for a sphere, and expansion of (3.16) to third order in $a k_{m}$ again yields (4.2) with shape parameters $\lambda_{f}=1$ and $\gamma_{f}=\frac{6}{5} \simeq 1.2$ (this value of the shape parameter $\gamma_{f}$, is consistent with that of the cut-off prescription). These values will be assumed from now on whenever we refer to the PL model. Accordingly, further discussion of the MFS model will henceforth be understood to correspond to the hard-sphere case.

\section{Remarks and discussion}

The main differences between the MFS model and exact results for a sphere $[1,23]$ are that

(i) the MFS matrix is of purely dipolar nature, while the exact matrix is not,

(ii) when $\mu_{s}=\mu_{m}$, the MFS $T$-matrix is proportional to the identity matrix, while the exact one distinguishes between longitudinal and transverse parts with respect to both $k_{1}$ and $k_{2}$; and

(iii) there is no dielectric-magnetic coupling between the dielectric and the magnetic polarizabilities (in consequence the MFS $T$-matrix cannot for example account for the eddy currents in a metal sphere).

Let us briefly examine causality. The PL model is not causal, as we can demonstrate via a simple example where $\varepsilon_{m}=1$ and $\varepsilon_{s}=2$. On physical grounds, the polarizability $\bar{\alpha}_{\varepsilon}$ should be a causal response function but, in the PL limit, one may readily verify that $\bar{\alpha}_{\varepsilon}$ 
possesses a spurious pole at $a k_{m}=2.32 \mathrm{i}$ in the upper complex $\omega$ half-plane ( $\omega$-UHP). This precludes the fulfilment of the Kramers-Kronig (KK) causality rules on this polarizability. For comparison, the physical resonance in this PL example is at $\left(a k_{m}\right)_{\varepsilon}=2.24$, i.e. with a modulus comparable with that of the spurious pole.

In order to illustrate the above point numerically, we have plotted in figure 1 the quantity $\operatorname{Re} \bar{\alpha}_{\varepsilon}$ against $a \omega / c$ for both the PL and the MFS model. The curves labelled (anal.) are obtained by directly computing $\operatorname{Re} \bar{\alpha}_{\varepsilon}$ from the expression for $\bar{\alpha}_{\varepsilon}$. The curves labelled (KK) are obtained via the KK formula

$$
\operatorname{Re}\left[\bar{\alpha}_{\varepsilon}(\omega)\right]=\lim _{\omega \rightarrow \infty}\left\{\operatorname{Re}\left[\bar{\alpha}_{\varepsilon}(\omega)\right]\right\}+\frac{2}{\pi} \mathcal{P} \int_{0}^{\infty} \frac{\omega^{\prime} \operatorname{Im}\left[\bar{\alpha}_{\varepsilon}\left(\omega^{\prime}\right)\right]}{\omega^{\prime 2}-\omega^{2}} \mathrm{~d} \omega^{\prime} .
$$

For frequency-dependent permittivities, realistic over the whole frequency range, the term $\lim _{\omega \rightarrow \infty}\left\{\operatorname{Re}\left[\bar{\alpha}_{\varepsilon}(\omega)\right]\right\}$ is necessarily zero (since physics requires that both $\varepsilon_{s}$ and $\varepsilon_{m}$ tend to the common limit 1). This limit can, however, be non-zero in general if $\varepsilon_{s}$ and $\varepsilon_{m}$ are assumed to be model values whose physical relevance is limited to a restricted frequency range.

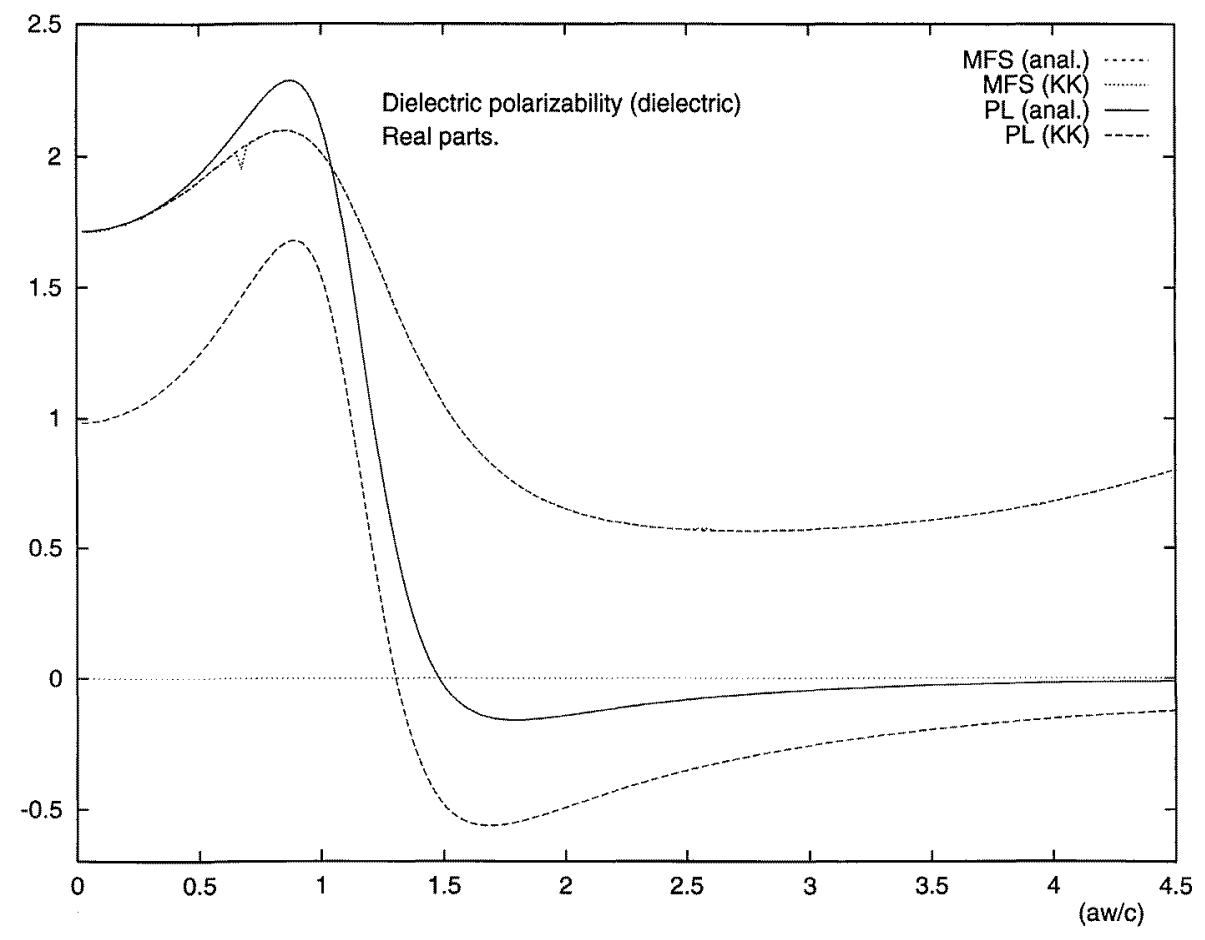

Figure 1. Tests of the KK relations carried out on the MFS and PL approximations for the dynamic polarizabilities of dielectric spheres, $\varepsilon_{s}=5$, embedded in a homogeneous background medium, $\varepsilon_{m}=1$. The curves MFS (anal.) and PL (anal.) are the real parts of the polarizabilities (equations (3.14)) calculated using equation (3.16) (MFS) and equation (4.2a) (PL), respectively. The curves MFS (KK) and PL (KK) for the real polarizabilities are calculated by applying the KK relation (5.1) to the imaginary parts of the polarizabilities.

Irrespective of the physical content of the models used for the constitutive parameters, equation (5.1) should always be satisfied. In the PL model, $\lim _{\omega \rightarrow \infty}\left\{\operatorname{Re}\left[\bar{\alpha}_{\varepsilon}(\omega)\right]\right\}$ is 0 
while, in the MFS model, $\lim _{\omega \rightarrow \infty}\left\{\operatorname{Re}\left[\bar{\alpha}_{\varepsilon}(\omega)\right]\right\}=\left(\varepsilon_{s}-\varepsilon_{m}\right) / \varepsilon_{s}$. Integral (5.1) has been numerically computed by combining the Cauchy principal value integration routine, and that for integration over a semi-infinite interval provided in the IMSL library package. In figure 1 , we have used $\varepsilon_{m}=1, \mu_{m}=1$ and $\varepsilon_{s}=5$. Both the direct and the KK calculations lead to the same result in the MFS case, except for a small deviation observable at $a \omega / c \simeq 0.675$ that is due to a breakdown of the integration method, but of no physical relevance (a different choice of the frequency step makes this discrepancy disappear). In contrast, the direct and KK curves fail to match in the PL model.

It is difficult to discuss the question of the poles in the polarizability in full generality for arbitrary frequency-dependent complex values of $\varepsilon_{m}$ and $\varepsilon_{s}$. We therefore restrict the following analytic discussion to the case where these quantities are real. This assumption implies that they should also be frequency independent and greater than 1 [20]. A purely imaginary pole in the $\omega$-UHP exists whenever the (real) equation

$$
\frac{\varepsilon_{s}}{\varepsilon_{m}}=\left.\left(1-\frac{1}{\bar{L}}\right)\right|_{\omega=\mathrm{i} x}
$$

admits a real solution $x$. This is always the case in the PL model, but it never happens in the MFS model provided that $\varepsilon_{s} / \varepsilon_{m}>0$, which is consistent with our above stated working hypothesis. Graphical investigations show that there is no other possibility for a pole in the $\omega$-UHP in the MFS model as long as $\operatorname{Re}\left(a k_{m}\right)>0\left(\operatorname{Im} \bar{L}=\operatorname{Re}\left(a k_{m}\right) \times\right.$ a purely positive function of $a k_{m}$ ).

We have also checked the KK rules numerically for several frequency-dependent models of permittivities (Drude metal models). In all cases under consideration, the plots of the direct and KK expressions for $\operatorname{Re} \bar{\alpha}_{\varepsilon}$ were nearly indistinguishable to the eye as in the frequency-independent case above.

We emphasize the fact that the $T$-matrix itself, $\mathbf{T}\left(k_{1} \mid k_{2}\right)$, properly normalized (its dielectric part has to be divided by $\left.(\omega / c)^{2}\right)$ obeys a similar KK formula when the Fourier momentum variables $k_{1}$ and $k_{2}$ are real frequency-independent quantities, but not if $k_{1}=k_{2}=k_{m}$ (on-shell values) for instance. Owing to the oscillatory shape factor $\overline{\psi_{0}}\left(k_{m}\right)^{2}$, $\mathbf{T}\left(k_{m} \mid k_{m}\right)$ is singular at $\omega=\mathrm{i} \infty$, and Jordan's lemma does not apply. This does not mean that causality is violated, but merely that the derivation of the KK formula, relying on contour integration, is no longer valid. The KK formula applied to $\mathbf{T}\left(k_{m} \mid k_{m}\right)$ has to be modified (via a transformation of the integrand) in order to yield correct results.

We now discuss the possibility of enriching the MFS model so as to account for dielectric-magnetic coupling. Quite interestingly, it turns out that under the 'renormalization' substitutions (the function $Q_{1}$ is defined in the appendix)

$$
\frac{1}{\varepsilon_{s}} \rightarrow Q_{1}\left(a k_{s}\right) \frac{1}{\varepsilon_{s}}+\left(1-Q_{1}\left(a k_{m}\right)\right) \frac{1}{\varepsilon_{m}} \quad \frac{1}{\mu_{s}} \rightarrow Q_{1}\left(a k_{s}\right) \frac{1}{\mu_{s}}+\left(1-Q_{1}\left(a k_{m}\right)\right) \frac{1}{\mu_{m}}
$$

the transverse-transverse ZZ and XX components (A9) of the vector spherical harmonics (VSH) expansion of the MFS $T$-matrix (cf. appendix) evaluated on shell, i.e. at $k_{1}=k_{2}=$ $k_{m}$, match the exact on-shell $l=1 \mathrm{ZZ}$ and XX components (A10) for a hard sphere [23]. Note that the substitutions preserve the unitarity identity, equation (3.4), since they involve only real quantities in the non-dissipative case. These substitutions somewhat generalize to arbitrary media and frequencies those proposed in $[24,25]$ in a slightly different context, which are

$$
\varepsilon_{s} \rightarrow \frac{\varepsilon_{s}}{Q_{1}\left(a k_{s}\right)} \quad \mu_{s} \rightarrow \frac{\mu_{s}}{Q_{1}\left(a k_{s}\right)} .
$$

The function $Q_{1}\left(a k_{s}\right)$ accounts for the spatial distribution of the inner fields and is responsible for the induced diamagnetic susceptibility due to eddy Foucault currents at 
the surface of a metallic sphere at low frequencies. For a metallic sphere in a dielectric medium, provided that $a k_{m} \ll 1 \ll a k_{s}$, one obtains $\bar{\alpha}_{\mu} \neq 0$ even if $\mu_{s}=\mu_{m}$. In this circumstance, equation (5.3) then reduces to equation (5.4). We remark that equation (5.4) may be directly obtained by solving the inner problem [20, 24, 25].

The problem with equation (5.3) or equation (5.4) is that neither substitution can be consistently performed in the MFS model when $k_{1}$ and $k_{2}$ are arbitrary. For instance, in order to recover the vanishing of certain terms when $\varepsilon_{s}=\varepsilon_{m}$ or $\mu_{m}=\mu_{s}$, displayed by the exact momentum-dependent $T$-matrix of a magnetic and dielectric sphere at $k_{1}=k_{2}=0$ (values of particular interest for defining the effective constitutive parameters of a random medium [23]). Moreover, the MFS model does not distinguish between the longitudinal and the transverse electric components of the $T$-matrix, so that a renormalization of the inner dielectric constant applies to all elements at once. In exact results, the dynamic behaviours of the transverse and longitudinal components are different. This cannot be corrected in the context of the present model because any $T$-matrix here has to be proportional to I when $k_{1}=k_{2}=0$.

In spite of its weaknesses, the MFS compares fairly well with the exact $l=1$ onshell formula (A10a), especially in the dissipative case: in figures 2(a) and 2(b), we plotted the real and imaginary parts of the MFS model, the PL model and equation (A10a) (normalized so as to yield the value of $\bar{\alpha}_{\varepsilon}$ when $\omega=0$ ), as a function of the reduced frequency. Figure 2(a) is a non-dissipative case: $\varepsilon_{s}=5, \varepsilon_{m}=1, \mu_{s}=\mu_{m}=1$. We see that the MFS model reproduces the exact dipolar formula up to the resonance region. The PL model, although overestimating the resonance height, yields a somewhat better overall shape. In the dissipative Drude case of figure 2(b), where we have arbitrarily put $\varepsilon_{s}=6-20 /\{(a \omega / c)[(a \omega / c)+0.035 \mathrm{i}]\}$, the MFS model is much closer to the exact dipolar result than is the PL model.

\section{Conclusion}

The mean-field spherical $T$-matrix that we introduced has several attractive features; it permits the consistent implementation of excluded volume effects in multiple-scattering models because of its separable structure, it obeys the unitarity identity, and finally it preserves causality for a wide class of physical constitutive parameters. The magneticdielectric coupling responsible for induced effective magnetism is, however, beyond its intrinsic abilities. A phenomenological renormalization of the inner dielectric constant corrects the situation when $k_{1}=k_{2}=k_{m}$, but cannot apply to the general off-shell case. The MFS model does not truly distinguish between transverse and longitudinal components. A simple and more physical model which would exhibit such a distinction seems hard to obtain without entering into the complications of the full problem. We believe, however, that the MFS model incorporates sufficient phenomenology to be useful in multiple-scattering applications.

\section{Acknowledgments}

This work has been performed under support of the CEA-Direction des Applications Militaires. One of us (Y-P P) thanks Dr B A van Tiggelen for pointing out to him the existence of [15]. We also thank Dr J-J Niez and Dr D Gogny for useful discussions. 

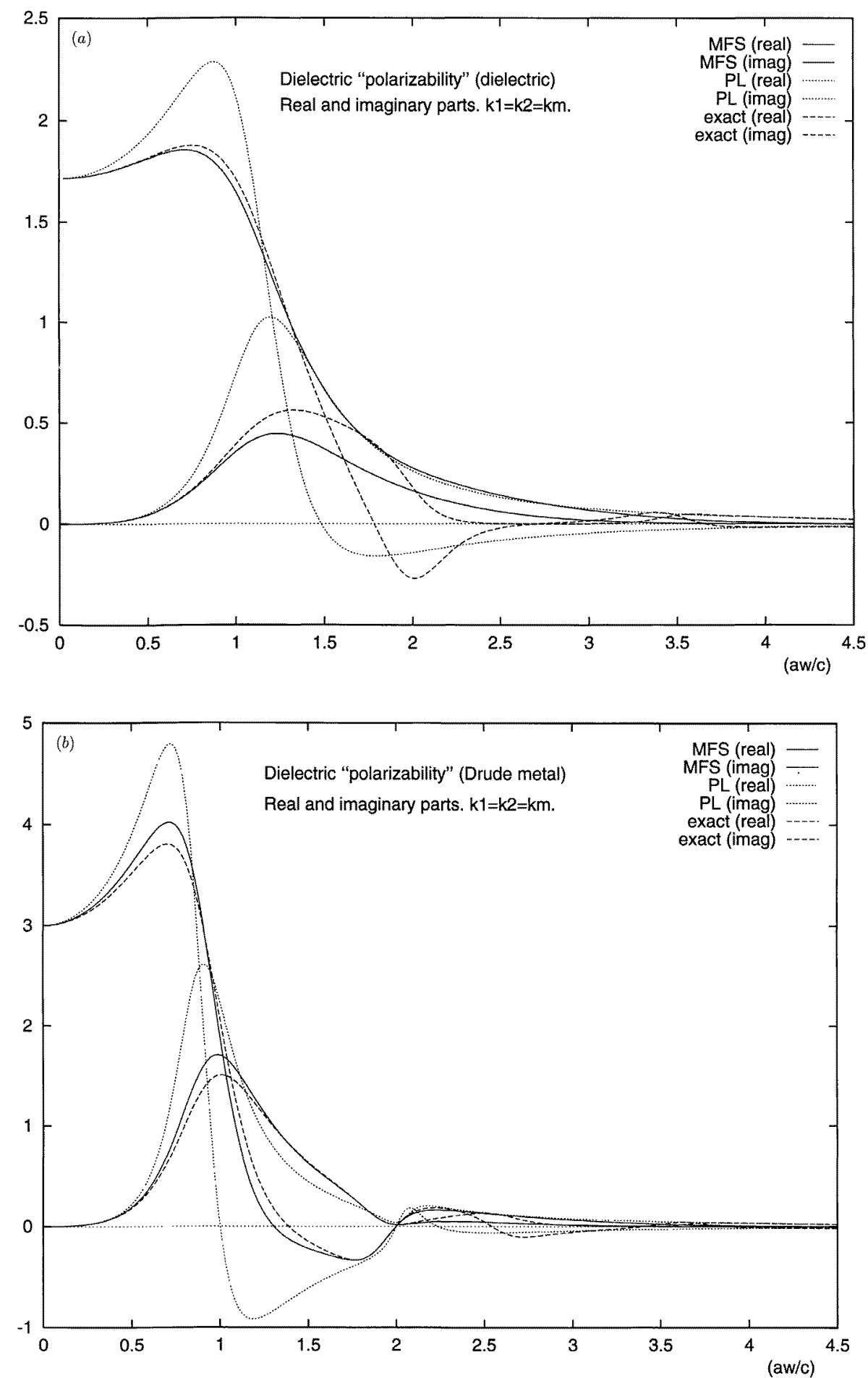

Figure 2. Comparison between the dynamic dielectric polarizabilities in the MFS and PL models compared with the exact dipolar on-shell polarizabilities deduced from the exact $T$-matrix values $T_{1}^{Z Z}\left(k_{m} \mid k_{m}\right)$ (see equation A10a): (a) non-dispersive scatterer, $\varepsilon_{s}=5, \varepsilon_{m}=1, \mu_{s}=\mu_{m}=1$; (b) dissipative Drude sphere, $\varepsilon_{m}=1, \varepsilon_{s}=6-20 /[(a \omega / c)(a \omega / c+0.035 i)], \mu_{s}=\mu_{m}=1$. 


\section{Appendix. Vector spherical harmonics}

In this work, we used orthonormalized spherical harmonics

$$
\int \mathrm{d} \Omega Y_{l m}(\Omega) Y_{l^{\prime} n^{\prime}}^{*}(\Omega)=\delta_{l, l^{\prime}} \delta_{n, n^{\prime}} .
$$

The vector spherical harmonics (VSHs) are the generalization for vector fields of the spherical harmonics for scalar fields. They are defined so as to provide an orthonormalized trihedral basis of definite angular momentum states $l \geqslant 0, n=-l, \ldots, l$ on which any vector field may be expanded, they are given by

$$
\begin{array}{ll}
\boldsymbol{N}_{l n}\left(\Omega_{k}\right) \equiv \boldsymbol{Y}_{l n}^{(0)}(\hat{\boldsymbol{k}}) \equiv \hat{\boldsymbol{k}} Y_{l n}\left(\Omega_{k}\right) & (l \geqslant 0) \\
\boldsymbol{Z}_{l n}\left(\Omega_{k}\right) \equiv \boldsymbol{Y}_{l n}^{(e)}(\hat{\boldsymbol{k}}) \equiv \frac{1}{\sqrt{l(l+1)}} \nabla_{\Omega_{k}} Y_{l n}\left(\Omega_{k}\right) & (l \geqslant 1) \\
\boldsymbol{X}_{l n}\left(\Omega_{k}\right) \equiv \boldsymbol{Y}_{l n}^{(m)}(\hat{\boldsymbol{k}}) \equiv \frac{1}{\sqrt{l(l+1)}} \hat{\boldsymbol{k}} \times \nabla_{\Omega_{k}} Y_{l n}\left(\Omega_{k}\right) & (l \geqslant 1)
\end{array}
$$

where the operator $\nabla_{\Omega_{k}}$ denotes the angular part of the operator $\nabla=\hat{k} \partial / \partial k+(1 / k) \nabla_{\Omega_{k}}$ in spherical coordinates. The more standard notation for the VSHs is $\boldsymbol{Y}_{l n}^{(0),(e),(m)}$, as used by Newton [27]. In the following, we employ the alternative VSH symbols $\boldsymbol{N}_{l n}, \boldsymbol{Z}_{l n}$ and $\boldsymbol{X}_{l n}$ as found in the book by Cohen-Tannoudji et al [26]. This alternative notation generalizes that of Jackson [28] and has the merit of rendering our formulae more readable by alleviating the need for superscripts.

The above VSHs comply with the orthonormalization conditions

$$
\int \mathrm{d} \Omega_{k} \boldsymbol{A}_{l n}^{*}\left(\Omega_{k}\right) \cdot \boldsymbol{B}_{l^{\prime} n^{\prime}}\left(\Omega_{k}\right)=\delta_{A, B} \delta_{l, l^{\prime}} \delta_{n, n^{\prime}}
$$

where $\boldsymbol{A}, \boldsymbol{B}$ represent $\boldsymbol{N}, \boldsymbol{X}$ or $\boldsymbol{Z}$ indifferently and satisfy the closure relation

$$
\sum_{l n}\left\{\left|N_{l n}\right\rangle\left\langle N_{l n}|+| X_{l n}\right\rangle\left\langle X_{l n}|+| Z_{l n}\right\rangle\left\langle Z_{l n}\right|\right\}=1 .
$$

Of use are the sums which follow from the identity

$$
\sum_{n} Y_{l n}\left(\Omega_{k_{1}}\right) Y_{l n}^{*}\left(\Omega_{k_{2}}\right)=\frac{2 l+1}{4 \pi} P_{l}(u)
$$

where $u=\hat{\boldsymbol{k}}_{1} \cdot \hat{\boldsymbol{k}}_{2}$ and $P_{l}$ is the Legendre polynomial given by

$$
P_{l}(x)=\frac{1}{2^{l} l !} \frac{\mathrm{d}^{l}}{\mathrm{~d} x^{l}}\left(x^{2}-1\right)^{l} .
$$

One finds that

$$
\begin{gathered}
\sum_{n} \boldsymbol{N}_{l n}\left(\Omega_{\boldsymbol{k}_{1}}\right) \boldsymbol{N}_{l n}^{*}\left(\Omega_{\boldsymbol{k}_{2}}\right)=\frac{2 l+1}{4 \pi} P_{l}(u) \hat{\boldsymbol{k}}_{1} \hat{\boldsymbol{k}}_{2} \\
\sum_{n} \boldsymbol{N}_{l n}\left(\Omega_{\boldsymbol{k}_{1}}\right) \boldsymbol{Z}_{l n}^{*}\left(\Omega_{\boldsymbol{k}_{2}}\right)=\frac{2 l+1}{4 \pi \sqrt{l(l+1)}} P_{l}^{\prime}(u) \hat{\boldsymbol{k}}_{1}\left[\hat{\boldsymbol{k}}_{1}-u \hat{\boldsymbol{k}}_{2}\right] \\
\sum_{n} \boldsymbol{Z}_{l n}\left(\Omega_{k_{1}}\right) \boldsymbol{N}_{l n}^{*}\left(\Omega_{\boldsymbol{k}_{2}}\right)=\frac{2 l+1}{4 \pi \sqrt{l(l+1)}} P_{l}^{\prime}(u)\left[\hat{\boldsymbol{k}}_{2}-u \hat{\boldsymbol{k}}_{1}\right] \hat{\boldsymbol{k}}_{2} \\
\sum_{n} \boldsymbol{Z}_{l n}\left(\Omega_{\boldsymbol{k}_{1}}\right) \boldsymbol{Z}_{l n}^{*}\left(\Omega_{\boldsymbol{k}_{2}}\right)=\frac{2 l+1}{4 \pi l(l+1)} P_{l}^{\prime \prime}(u)\left[\hat{\boldsymbol{k}}_{2}-u \hat{\boldsymbol{k}}_{1}\right]\left[\hat{\boldsymbol{k}}_{1}-u \hat{\boldsymbol{k}}_{2}\right]
\end{gathered}
$$




$$
\begin{gathered}
+\frac{2 l+1}{4 \pi l(l+1)} P_{l}^{\prime}(u)\left[\mathbf{I}-\hat{\boldsymbol{k}}_{1} \hat{\boldsymbol{k}}_{1}-\hat{\boldsymbol{k}}_{2} \hat{\boldsymbol{k}}_{2}+u \hat{\boldsymbol{k}}_{1} \hat{\boldsymbol{k}}_{2}\right] \\
\sum_{n} \boldsymbol{X}_{l n}\left(\Omega_{\boldsymbol{k}_{1}}\right) \boldsymbol{X}_{l n}^{*}\left(\Omega_{\boldsymbol{k}_{2}}\right)=\frac{2 l+1}{4 \pi l(l+1)} P_{l}^{\prime \prime}(u)\left(\hat{\boldsymbol{k}}_{1} \times \hat{\boldsymbol{k}}_{2}\right)\left(\hat{\boldsymbol{k}}_{2} \times \hat{\boldsymbol{k}}_{1}\right) \\
+\frac{2 l+1}{4 \pi l(l+1)} P_{l}^{\prime}(u)\left[u \mathbf{I}-\hat{\boldsymbol{k}}_{2} \hat{\boldsymbol{k}}_{1}\right] .
\end{gathered}
$$

These formulae permit us to deduce the representations

$$
\begin{aligned}
\mathbf{I}=\frac{4 \pi}{3} \sum_{n}\left[N_{1 n}\left(\Omega_{k_{1}}\right) N_{1 n}^{*}\left(\Omega_{k_{2}}\right)+\sqrt{2} N_{1 n}\left(\Omega_{k_{1}}\right) Z_{1 n}^{*}\left(\Omega_{k_{2}}\right)\right. \\
\left.+\sqrt{2} \boldsymbol{Z}_{1 n}\left(\Omega_{k_{1}}\right) \boldsymbol{N}_{1 n}^{*}\left(\Omega_{k_{2}}\right)+2 \boldsymbol{Z}_{1 n}\left(\Omega_{k_{1}}\right) \boldsymbol{Z}_{1 n}^{*}\left(\Omega_{k_{2}}\right)\right] \\
-\hat{\boldsymbol{k}}_{1} \times \hat{\boldsymbol{k}}_{2} \times=\frac{8 \pi}{3} \sum_{n} \boldsymbol{X}_{1 n}\left(\Omega_{k_{1}}\right) \boldsymbol{X}_{1 n}^{*}\left(\Omega_{k_{2}}\right) .
\end{aligned}
$$

The expansion of the MFS $T$-matrix over the vector spherical harmonics basis is

$$
\mathbf{T}_{\mathbf{0}}\left(\boldsymbol{k}_{1} \mid \boldsymbol{k}_{2}\right)=\sum_{\substack{l, n \\ A, B=N, Z, X}} T_{l}^{A B}\left(k_{1} \mid k_{2}\right) \boldsymbol{A}_{l n}\left(\Omega_{\boldsymbol{k}_{1}}\right) \boldsymbol{B}_{l n}^{*}\left(\Omega_{k_{2}}\right)
$$

with

$$
\begin{aligned}
T_{l}^{N X}\left(k_{1} \mid k_{2}\right) & =T_{l}^{X N}\left(k_{1} \mid k_{2}\right)=T_{l}^{Z X}\left(k_{1} \mid k_{2}\right)=T_{l}^{X Z}\left(k_{1} \mid k_{2}\right)=0 \\
T_{l}^{Z Z}\left(k_{1} \mid k_{2}\right) & =\delta_{l, 1} \frac{8 \pi}{3} V_{s} \frac{k_{m}^{2}}{\mu_{m}} \bar{\alpha}_{\varepsilon} \overline{\psi_{0}}\left(k_{1}\right) \overline{\psi_{0}}\left(k_{2}\right) \\
& =2 T_{l}^{N N}\left(k_{1} \mid k_{2}\right)=\sqrt{2} T_{l}^{N Z}\left(k_{1} \mid k_{2}\right)=\sqrt{2} T_{l}^{Z N}\left(k_{1} \mid k_{2}\right) \\
T_{l}^{X X}\left(k_{1} \mid k_{2}\right) & =\delta_{l, 1} \frac{8 \pi}{3} V_{s} \frac{k_{1} k_{2}}{\mu_{m}} \bar{\alpha}_{\mu} \overline{\Psi_{0}}\left(k_{1}\right) \overline{\psi_{0}}\left(k_{2}\right)
\end{aligned}
$$

thus illustrating its dipolar nature.

For comparison, the exact on-shell $\left(k_{1}=k_{2}=k_{m}\right)$ transverse-transverse elements ZZ and $\mathrm{XX}$ for a sphere are $(l \geqslant 1)[23]$

$$
\begin{aligned}
& \frac{(2 \pi)^{3}}{V_{s}} \frac{\mu_{m}}{k_{m}^{2}} T_{l}^{Z Z}\left(k_{m} \mid k_{m}\right)=4 \pi(l+1) \frac{\varepsilon_{s} Q_{l}\left(a k_{m}\right)-\varepsilon_{m} Q_{l}\left(a k_{s}\right)}{l \varepsilon_{s} Q_{l}^{(1)}\left(a k_{m}\right)+(l+1) \varepsilon_{m} Q_{l}\left(a k_{s}\right)} \frac{3 j_{l}\left(a k_{m}\right)}{\mathrm{i}\left(a k_{m}\right)^{3} h_{l}^{(1)}\left(a k_{m}\right)} \\
& \frac{(2 \pi)^{3}}{V_{s}} \frac{\mu_{m}}{k_{m}^{2}} T_{l}^{X X}\left(k_{m} \mid k_{m}\right)=4 \pi(l+1) \frac{\mu_{s} Q_{l}\left(a k_{m}\right)-\mu_{m} Q_{l}\left(a k_{s}\right)}{l \mu_{s} Q_{l}^{(1)}\left(a k_{m}\right)+(l+1) \mu_{m} Q_{l}\left(a k_{s}\right)} \frac{3 j_{l}\left(a k_{m}\right)}{\mathrm{i}\left(a k_{m}\right)^{3} h_{l}^{(1)}\left(a k_{m}\right)}
\end{aligned}
$$

where $V_{s}=4 \pi a^{3} / 3$ is the volume of the sphere. These are simply the well known Mie coefficients deduced from the solution of the outer problem [29].

The functions $Q_{l}$ and $Q_{l}^{(1)}$ given by

$$
\begin{aligned}
& Q_{l}(x)=\frac{1}{l+1} \frac{\left[x j_{l}(x)\right]^{\prime}}{j_{l}(x)} \\
& Q_{l}^{(1)}(x)=-\frac{1}{l} \frac{\left[x h_{l}^{(1)}(x)\right]^{\prime}}{h_{l}^{(1)}(x)}
\end{aligned}
$$


are defined in terms of the spherical Bessel and Hankel functions $j_{l}$ and $h_{l}^{(1)}$ so that they go to 1 in the limit $x \rightarrow 0$. They obey the Wronskian identity

$$
l Q_{l}^{(1)}(x)+(l+1) Q_{l}(x)=\frac{1}{\mathrm{i} x j_{l}(x) h_{l}^{(1)}(x)} .
$$

For $l=1$ we find that

$$
\begin{aligned}
& Q_{1}(x)=\frac{1}{2}\left[-1+\frac{x^{2}}{1-x \cot (x)}\right] \\
& Q_{1}^{(1)}(x)=1-\frac{x^{2}}{1-\mathrm{i} x} .
\end{aligned}
$$

\section{References}

[1] Tsang L and Kong J A 1980 J. Appl. Phys. 513465

[2] Tsang L and Kong J A 1981 J. Appl. Phys. 525448

[3] Kirkpatrick T R 1985 Phys. Rev. B 315746

[4] Sheng P 1995 Introduction to Wave Scattering, Localization and Mesoscopic Phenomena (New York: Academic), and references therein

[5] Garland J C and Tanner D B (ed) 1978 Proc. 1st ETOPIM (Ohio State University, 1977) (New York: American Institute of Physics)

Lafait J and Tanner D B (ed) 1989 Physica A 157

[6] Lax M 1951 Rev. Mod. Phys. 23287

[7] Frisch U 1968 Probabilistic Methods in Applied Mathematics vol 1 (New York: Academic) pp 75-198

[8] Ishimaru A 1978 Wave Propagation and Scattering in Random Media vol 2 (New York: Academic)

[9] Lagendijk A D and van Tiggelen B A 1996 Phys. Rep. 270143

[10] Balian R and Niez J-J 1995 J. Physique I 57

[11] Jing X, Sheng P and Zhou M 1992 Phys. Rev. A 466513

[12] Henrici P 1988 Applied and Computational Complex Analysis vol 1 (New York: Wiley)

[13] Pellegrini Y-P, Thibaudeau P and Stout D B 1996 Proc. Journées Maxwell '95 (Bordeaux-Lac, 1996) (ed) J-F Eloy (Le Barp: CEA-CESTA)

[14] van Tiggelen B A, Lagendijk A and Tip A 1990 J. Phys.: Condens. Matter 27653

[15] Nieuwenhuizen TM, Lagendijk A and van Tiggelen B 1992 Phys. Lett. A 169191

[16] Joachain C J 1975 Quantum Collision Theory (Amsterdam: North-Holland)

[17] The condition $\hat{\boldsymbol{n}} \times \boldsymbol{H}_{1}=\hat{\boldsymbol{n}} \times \boldsymbol{H}_{2}$ may be traced to the appearance of two different momentum variables $\boldsymbol{k}_{1}$ and $k_{2}$ in the double-vector product of equation (2.7). In performing the Fourier transform of the scattering potential (2.3), which is an operator (besides being a function of two variables), one must sandwich it between a factor $\exp \left(-\mathrm{i} \boldsymbol{r} \cdot \boldsymbol{k}_{1}\right)$ on the left, and a factor $\exp \left(\mathrm{i} \boldsymbol{r}^{\prime} \cdot \boldsymbol{k}_{2}\right)$ on the right in order to recover this correct double-vector product.

[18] Botet R, Ranou P and Cabane M 1995 J. Phys. A: Math. Gen. 28 297, and references therein

[19] Berthier S 1988 Ann. Phys., Paris 13503

[20] Landau L, Lifchitz E and Pitaevskii LP 1984 Electrodynamics of Continuous Media (Oxford: Pergamon)

[21] Pellegrini Y-P, Thibaudeau P and Stout D B 1995 Waves and Heterogeneous Media (Les Houches, 21-31 March 1995) unpublished

[22] Parisi G 1988 Statistical Field Theory (New York: Addison-Wesley)

[23] Pellegrini Y-P, Thibaudeau P and Stout D B 1996 to be published

[24] Rousselle D, Berthault A, Acher O, Bouchaud J P and Zérah P G 1993 J. Appl. Phys. 74475

[25] Lagarkov A N, Sarychev A K, Smychkovich Y R and Vinogradov A P 1992 J. Electr. Waves Appl. 61159

[26] Cohen-Tannoudji C, Dupont-Roc J and Grynberg G 1987 Introduction à l'Electrodynamique Quantique (Paris: InterEditions-Editions du CNRS)

[27] Newton R G 1966 Scattering Theory of Waves and Particles (New York: McGraw-Hill)

[28] Jackson J D 1975 Classical Electrodynamics (New York: Wiley)

[29] Bohren C F and Huffman D R 1983 Absorption and Scattering of Light by Small Particles (New York: Wiley) 